\title{
Use of angiotensin-converting enzyme inhibitors and angiotensin II receptor blockers in context of COVID-19 outbreak: a retrospective analysis
}

\begin{abstract}
Jiuyang $\mathrm{Xu}^{1, *}$, Chaolin Huang ${ }^{2, *}$, Guohui Fan ${ }^{3,4,5, *}$, Zhibo Liu ${ }^{3,4, *}$, Lianhan Shang ${ }^{3,4,6}$, Fei Zhou ${ }^{3,4}$, Yeming Wang ${ }^{3,4,7}$, Jiapei $\mathrm{Yu}^{1}$, Luning Yang ${ }^{1}$, Ke Xie ${ }^{3,7}$, Zhisheng Huang ${ }^{3,8}$, Lixue Huang ${ }^{3,7}$, Xiaoying Gu, ${ }^{3,4,5}$, Hui Li ${ }^{3,4}$, Yi Zhang ${ }^{3,4}$, Yimin Wang ${ }^{3,4}$, Frederick G. Hayden ${ }^{9}$, Peter W. Horby ${ }^{10}$, Bin Cao $(\bowtie)^{3,4,7,11}$, Chen Wang $(\bowtie)^{3,4,11,12}$

${ }^{I}$ Department of Basic Medical Sciences, Tsinghua University School of Medicine, Beijing 100084, China; ${ }^{2}$ Department of Infectious Diseases, Jinyintan Hospital, Wuhan 430030, China; ${ }^{3}$ Department of Pulmonary and Critical Care Medicine, Center of Respiratory Medicine, National Clinical Research Center for Respiratory Diseases, China-Japan Friendship Hospital, Beijing 100029, China; ${ }^{4}$ Institute of Respiratory Medicine, Chinese Academy of Medical Sciences and Peking Union Medical College, Beijing 100730, China; ${ }^{5}$ Institute of Clinical Medical Sciences, China-Japan Friendship Hospital, Beijing 100029, China; ${ }^{6}$ Beijing University of Chinese Medicine, Beijing 100029, China; ${ }^{7}$ Department of Respiratory Medicine, Capital Medical University, Beijing 100029, China; ${ }^{8}$ Graduate School of Peking Union Medical College, Chinese Academy of Medical Sciences, Beijing 100730, China; ${ }^{9}$ Department of Medicine, University of Virginia School of Medicine, Charlottesville, VA 22904, USA; ${ }^{10}$ Centre for Tropical Medicine and Global Health, University of Oxford, Oxford OXI 2JD, UK, ${ }^{11}$ Tsinghua University-Peking University Joint Center for Life Sciences, Beijing 100084, China; ${ }^{2}$ Chinese Academy of Engineering, Beijing 100088, China
\end{abstract}

(C) Higher Education Press 2020

Abstract The possible effects of angiotensin-converting enzyme inhibitors (ACEIs) or angiotensin II receptor blockers (ARBs) on COVID-19 disease severity have generated considerable debate. We performed a single-center, retrospective analysis of hospitalized adult COVID-19 patients in Wuhan, China, who had definite clinical outcome (dead or discharged) by February 15, 2020. Patients on anti-hypertensive treatment with or without ACEI/ARB were compared on their clinical characteristics and outcomes. The medical records from 702 patients were screened. Among the 101 patients with a history of hypertension and taking at least one anti-hypertensive medication, 40 patients were receiving ACEI/ARB as part of their regimen, and 61 patients were on antihypertensive medication other than ACEI/ARB. We observed no statistically significant differences in percentages of in-hospital mortality $(28 \%$ vs. $34 \%, P=0.46)$, ICU admission $(20 \%$ vs. $28 \%, P=0.37)$ or invasive mechanical ventilation $(18 \%$ vs. $26 \%, P=0.31)$ between patients with or without ACEI/ARB treatment. Further multivariable adjustment of age and gender did not provide evidence for a significant association between ACEI/ARB treatment and severe COVID-19 outcomes. Our findings confirm the lack of an association between chronic receipt of reninangiotensin system antagonists and severe outcomes of COVID-19. Patients should continue previous antihypertensive therapy until further evidence is available.

Keywords COVID-19; SARS-CoV-2; hypertension; angiotensin-converting enzyme inhibitor; angiotensin II receptor blocker

\section{Introduction}

The coronavirus disease 2019 (COVID-19) outbreak has

Received May 26, 2020; accepted June 3, 2020

Correspondence: Bin Cao, caobin_ben@163.com; Chen Wang, wangchen@pumc.edu.cn

${ }^{*}$ These authors contributed equally to this study. spread across the world with more than 5.1 million confirmed cases [1]. Early reports on clinical characteristics have identified hypertension as the most common comorbidity reported in COVID-19 patients [2-5]. Zhou et al. documented pre-existing hypertension as a risk factor for in-hospital death of COVID-19 [6]. This observation is of great interest since the renin-angiotensin system (RAS) is an important component in regulating blood pressure, and the transmembrane angiotensin-converting enzyme 2 
(ACE2), which catalyzes the cleavage of angiotensin II (a vasoconstrictor) into vasodilatory peptides, is also the cellular entry receptor for SARS-CoV-2.

Given the common use of angiotensin-converting enzyme inhibitors (ACEIs) and angiotensin II receptor blockers (ARBs) in patients with cardiovascular diseases [7], there has been wide discussion whether administration of ACEI/ARB might modulate COVID-19 disease severity. Concerns have been raised whether hypertensive patients should stop taking $\mathrm{ACEI} / \mathrm{ARB}$ and switch to other anti-hypertensive drugs in the context of COVID-19 pandemic $[8,9]$. There have also been counter-arguments in favor of the use of ACEI/ARB in COVID-19 patients, based on previous studies showing that ACEI/ARB are associated with improvements in pneumonia-related outcomes $[10,11]$, as well as a protective role of ARBs in SARS-CoV and influenza infected animals $[12,13]$.

The renin-angiotensin system (RAS) plays a central role in regulating blood pressure and maintaining hemodynamic homeostasis. In hypovolemic conditions, angiotensinogen is serially cleaved by renin and angiotensinconverting enzyme (ACE) into angiotensin I (Ang I) and angiotensin II (Ang II), respectively. Ang II then acts on the cellular receptor angiotensin II receptor I (AT1) to constrict the blood vessels and increases blood pressure (Fig. 1). To counteract this vaso-constrictive effect of RAS,

A

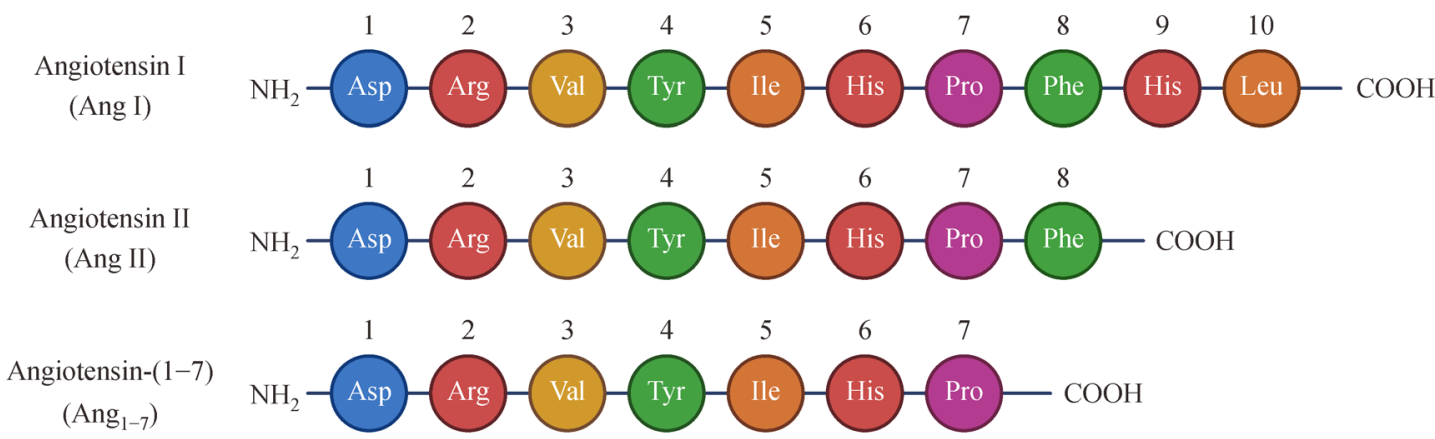

B

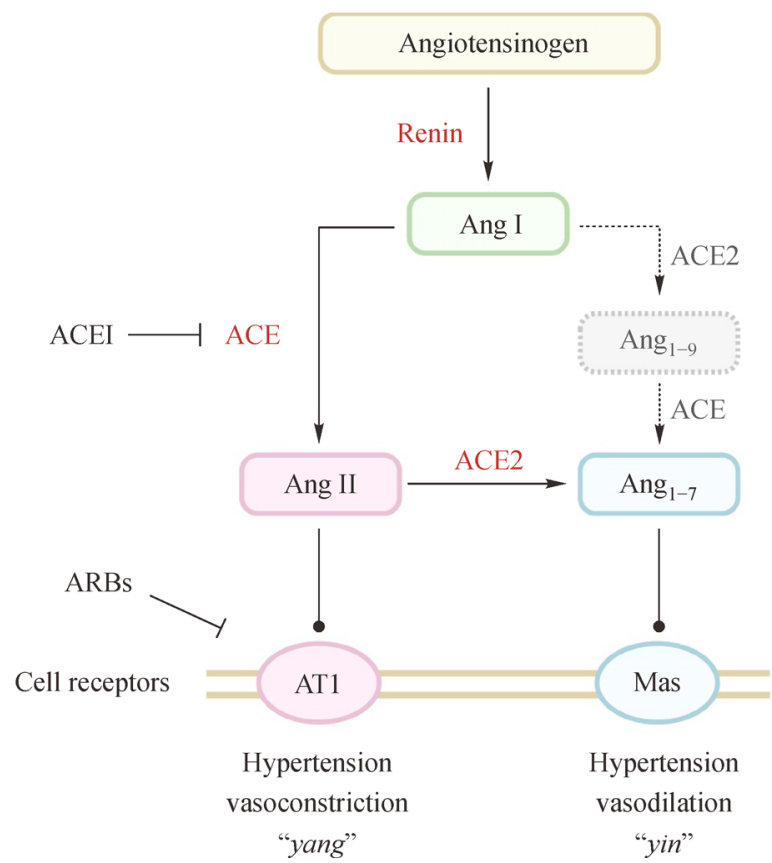

Fig. 1 Physiology of ACE/AngII/AT1 and ACE2/Ang $1_{-7} /$ Mas axes. (A) Schematic structures of angiotensin and its derivatives. The three-letter codes were used to represent amino acid residues. (B) Physiology of angiotensin processing and blood regulation. In hypovolemic conditions, renin, secreted by cells in macula densa in the kidney, cleaves angiotensinogen into a decapeptide angiotensin I (Ang I). The angiotensin-converting enzyme (ACE) further cleaves the peptide into an octapeptide angiotensin II (Ang II), which acts on the cellular receptor angiotensin II receptor I (AT1) to constrict the blood vessels and increases blood pressure ("yang"). ACE2 is a monocarboxypeptidase transforming the Ang II into angiotensin-(1-7) $\left(\mathrm{Ang}_{1-7}\right)$, which binds to the G protein-coupled receptor Mas to dilate the blood vessels and counter-balance ACE/Ang II in blood pressure regulation (“yin"). Figure was generated with BioRender. 
the human body has developed another system in an exquisite "yin-yang" balance. The ACE2 is a homolog of ACE $[14,15]$ that metabolizes Ang II into angiotensin-(17) $\left(\mathrm{Ang}_{1-7}\right)$, which dilates the blood vessels and counterbalance ACE/Ang II in blood pressure regulation [16] (Fig. 1). Of note, in a rat model of lipopolysaccharide-induced acute respiratory distress syndrome (ARDS), reduced pulmonary levels of $\mathrm{Ang}_{1-7}$ contribute to disease pathogenesis, and administration of this peptide or the ARB losartan reduces the development of ARDS [17]. The nonangiotensinase function of ACE2 was recognized when it was identified to be cellular receptor for severe acute respiratory syndrome coronavirus (SARS-CoV) [18], as well as more recently for SARS-CoV-2 [19-21]. ACE2 expression has been documented in various human organs, including oral and nasal mucosa, lung, intestine, kidney, heart, arterial/venous endothelium and others [22-25].

High-quality clinical evidence for ACEI/ARB usage in COVID-19 patients is insufficient to draw definitive conclusions whether chronic ACEI/ARB should be continued or stopped [26]. Several recent retrospective, hospital-based studies have reported no adverse or marginal protective effect of ACEI/ARB on the clinical outcomes of COVID-19 patients [27-32]. To contribute to this discussion, we have performed a secondary analysis on a previously-reported single-center retrospective cohort of COVID-19 patients in Wuhan, China [6].

\section{Materials and methods}

\section{Study design and data collection}

We previously collected data from 702 hospitalized adult COVID-19 patients admitted to Jinyintan Hospital (Wuhan, China), who had definite outcome (dead or discharged) between December 29, 2019 and February 15, 2020 [6]. Data were extracted from medical records into an electronic data collection form by two independent researchers. Their electronic medical records were screened for medical history of hypertension in past medical history or admitting diagnosis and for oral antihypertensive treatment before and during hospitalization. The anti-hypertensive treatments we included in the analysis were ACEI, ARB, $\beta$-blockers, calcium channel blockers (CCBs), diuretics, and others ( $\alpha$-blockers and traditional Chinese medicine). Patients with definitive long-term anti-hypertensive treatments, as either recorded in the medical history or in the prescribed medication chart as standing order during hospitalization, were included in the final analysis. The comorbidities were either selfreported by the patients or evaluated by attending physicians and recorded in admitting diagnosis.

The study was approved by the Research Ethics Commission of Jinyintan Hospital (KY-2020-01.01) and the informed consent was waived by the Research Ethics Commission.

\section{Definitions}

Fever was defined as axillary temperature of at least $37.3^{\circ} \mathrm{C}$. Sepsis and septic shock were evaluated based on Sepsis-3 International Consensus [33]. ARDS was diagnosed according to the Berlin Definition [34]. Acute kidney injury was defined according to the KDIGO clinical practice guidelines [35]. Diagnosis of acute cardiac injury was made if serum levels of high-sensitive cardiac troponin I was above the 99th percentile upper reference limit [36]. Secondary infection, hypoproteinemia, and coagulopathy were defined as previously [6].

\section{Statistical analysis}

Continuous variables were expressed as median (interquartile range (IQR)) and categorical variables as number (proportion). Two-group comparisons (ACEI/ARB vs. non-ACEI/ARB) were conducted with Mann-Whitney U test or $\chi^{2} /$ Fisher exact test where appropriate. The risk of death, ICU admission, invasive mechanical ventilation and corresponding odds ratio both with and without adjustment for age and gender were calculated by Logistic model, comparing patients in ACEI/ARB group with those in nonACEI/ARB group. Four previously-reported risk factors for death (age, history of diabetes, qSOFA score, and Ddimer) were included in univariable and multivariable analysis with Logistic model. A 2-sided $\alpha$ less than 0.05 was considered statistically significant. Statistical analyses were conducted using SAS software (version 9.4, SAS Institute) and SPSS software (version 25, IBM).

\section{Results}

\section{Patients and clinical characteristics}

A total of 702 adult laboratory-confirmed COVID-19 patients admitted to Jinyintan Hospital, who had definite clinical outcome by February 15, 2020, were screened in this study, of whom 188 patients had a medical history of hypertension. Among them, 101 patients had antihypertensive treatment medications recorded in their medical records and were included in the final analysis. The schematic of patient selection is shown in Fig. 2. Patients who took ACEI or ARB as part of their antihypertensive therapy were included in the ACEI/ARB cohort $(n=40)$, and those without were categorized in the non-ACEI/ARB cohort $(n=61)$.

Patients in ACEI/ARB group and non-ACEI/ARB group were comparable in age (median, 66.5 vs. 65.0 years, $P=0.80$ ) and gender distribution (male, $48 \%$ vs. 


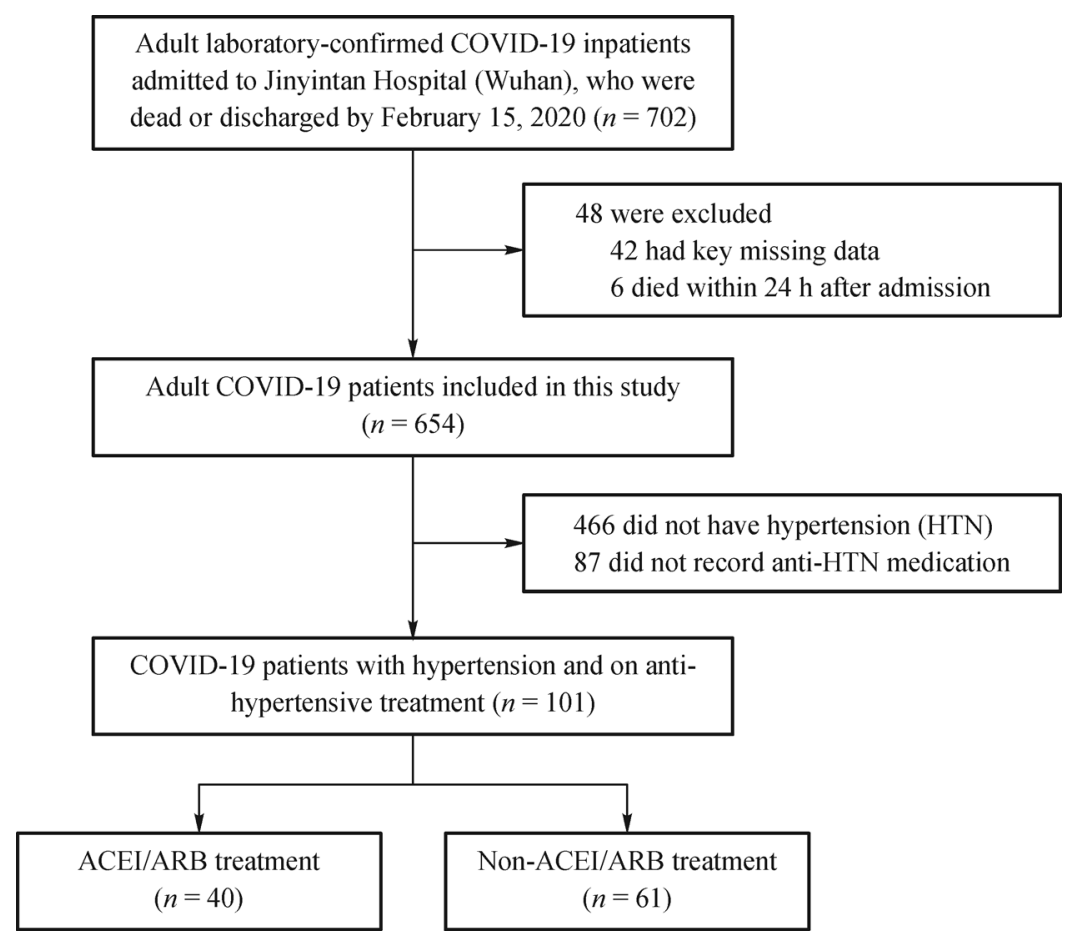

Fig. 2 Schematic for patient selection in this study.

$56 \%, P=0.42$ ), as shown in Table 1 . They were also in similar phase of the disease with median time from symptom onset to admission being 12.5 days (IQR 8.516.0 days) and 13.0 days (IQR 8.5-15.5 days), respectively. In addition to hypertension, patients in two groups also shared similar proportions of comorbidities and smoking history.

The vital signs and laboratory test results on admission were generally similar between the two groups (Table 1). Comparable proportions of patients in two groups had lymphocyte count less than $0.8 \times 10^{9} / \mathrm{L}(55 \%$ vs. $48 \%, P$ $=0.51)$, increased serum cardiac troponin I ( $18 \%$ vs. $19 \%$, $P=0.88)$, and increased serum D-dimer levels (59\% vs. $58 \%, P=0.95)$. The percentages of heart failure, defined as brain natriuretic peptide (BNP) value $\geqslant 100 \mathrm{pg} / \mathrm{mL}$ on admission, were also similar among the two groups (28\% vs. $27 \%, P=0.93$ ). Values of the inflammatory markers, including serum ferritin, procalcitonin, and interleukin-6 were not different between the two groups, nor were chest imaging features distinguishable. The sequential organ failure assessment (SOFA) scores in ACEI/ARB group (median 2.0, IQR 1.0-4.0) were numerically smaller than those in non-ACEI/ARB group (median 4.0, IQR 2.0-4.5), although the difference is not statistically significant $(P=$ $0.29)$.

\section{Anti-hypertensive treatment}

Among the 101 patients recorded with anti-hypertensive medication, 40 cases had therapies based on ACEI/ARB, either as mono-therapy or combined with $\beta$-blockers, $\mathrm{CCB}$, thiazide diuretics or spironolactone (Tables 2 and 3 ). No usage of sacubitril-valsartan was reported in this cohort. The non-ACEI/ARB therapy was mostly based on calcium channel blockers (Tables 2 and 3). The usage of other anti-hypertensive medications ( $\alpha$-blocker, $\beta$-blocker, and diuretics) was comparable between the two groups (Table 2). Similar percentages of patients were under optimal blood pressure control based on the vital signs taken on admission (Table 1). Chronic treatment on RAS antagonists was not associated with statistically significant changes in serum electrolyte (sodium and potassium) or serum creatinine levels (Table 1).

\section{Association between ACEI/ARB usage with severe clinical outcome of COVID-19}

Compared with the hypertensive patients who were not given ACEI/ARB, we observed no significant differences in percentages of in-hospital mortality $(28 \%$ vs. $34 \%, P=$ 0.46 ), ICU admission ( $20 \%$ vs. $28 \%, P=0.37$ ), or mechanical ventilation ( $18 \%$ vs. $26 \%, P=0.31)$ in those receiving a regimen that included an ACEI or ARB. Of note, the percentages with these major outcomes were uniformly smaller in ACEI/ARB group. Similarly, numerically fewer patients in ACEI/ARB group developed respiratory failure (43\% vs. $59 \%, P=0.10)$, sepsis $(48 \%$ vs. $59 \%, P=0.26)$ or ARDS ( $28 \%$ vs. $36 \%, P=0.37)$ 
Table 1 Demographic, clinical, laboratory, and radiologic findings on admission

\begin{tabular}{|c|c|c|c|c|}
\hline Characteristics & $\begin{array}{l}\text { Total } \\
n=101\end{array}$ & $\begin{array}{l}\mathrm{ACEI} / \mathrm{ARB} \\
n=40\end{array}$ & $\begin{array}{l}\text { Non-ACEI/ARB } \\
n=61\end{array}$ & $P$ value \\
\hline Age (year) & $65.0(58.0,73.0)$ & $66.5(58.0,72.0)$ & $65.0(58.0,74.0)$ & 0.8024 \\
\hline Gender, male, $n(\%)$ & $53(52)$ & $19(48)$ & $34(56)$ & 0.4175 \\
\hline Smoking, $n(\%)$ & $4(4)$ & $3(8)$ & $1(2)$ & 0.3394 \\
\hline $\begin{array}{l}\text { Time from illness onset to } \\
\text { admission (day) }\end{array}$ & $13.0(8.5,16.0)$ & $12.5(8.5,16.0)$ & $13.0(8.5,15.5)$ & 0.7805 \\
\hline \multicolumn{5}{|l|}{ Comorbidity } \\
\hline Hypertension, $n(\%)$ & $101(100)$ & $40(100)$ & $61(100)$ & NA \\
\hline Diabetes, $n(\%)$ & $19(19)$ & $8(20)$ & $11(18)$ & 0.8046 \\
\hline Heart failure, $n(\%)$ & $1(1)$ & $0(0)$ & $1(2)$ & 1.0000 \\
\hline Coronary heart disease, $n(\%)$ & $12(12)$ & $5(13)$ & $7(11)$ & 1.0000 \\
\hline COPD, $n(\%)$ & $2(2)$ & $1(3)$ & $1(2)$ & 1.0000 \\
\hline Carcinoma, $n(\%)$ & $5(5)$ & $2(5)$ & $3(5)$ & 1.0000 \\
\hline Chronic kidney disease, $n(\%)$ & $2(2)$ & $0(0)$ & $2(3)$ & 0.5168 \\
\hline \multicolumn{5}{|l|}{ Vital signs } \\
\hline Respiratory rate $>24 / \mathrm{min}, n(\%)$ & $19(19)$ & $6(15)$ & $13(21)$ & 0.4273 \\
\hline Systolic BP (mmHg) & $135(122,152)$ & $138(131,152)$ & $134(123,147)$ & 0.6617 \\
\hline$\geqslant 140, n(\%)$ & $39(39)$ & $17(43)$ & $22(36)$ & 0.5160 \\
\hline Diastolic BP (mmHg) & $81(75,88)$ & $79(74,90)$ & $82(76,87)$ & 0.4222 \\
\hline$\geqslant 90 \mathrm{mmHg}, n(\%)$ & $22(22)$ & $11(28)$ & $11(18)$ & 0.2596 \\
\hline \multicolumn{5}{|l|}{ Symptoms } \\
\hline Fever, $n(\%)$ & $88(87)$ & $31(78)$ & $57(93)$ & 0.0193 \\
\hline Cough, $n(\%)$ & $80(79)$ & $30(75)$ & $50(82)$ & 0.3988 \\
\hline Sputum, $n(\%)$ & $30(30)$ & $11(28)$ & $19(31)$ & 0.6948 \\
\hline Myalgia, $n(\%)$ & $9(9)$ & $2(5)$ & $7(11)$ & 0.4472 \\
\hline Headache, $n(\%)$ & $9(9)$ & $5(13)$ & $4(7)$ & 0.5040 \\
\hline Fatigue, $n(\%)$ & $39(39)$ & $15(38)$ & $24(39)$ & 0.8523 \\
\hline Diarrhea, $n(\%)$ & $8(8)$ & $3(8)$ & $5(8)$ & 1.0000 \\
\hline Dyspnea, $n(\%)$ & $64(63)$ & $23(58)$ & $41(67)$ & 0.3217 \\
\hline \multicolumn{5}{|l|}{ Laboratory findings } \\
\hline $\mathrm{Na}(\mathrm{mmol} / \mathrm{L})$ & $140.0(139.0,142.0)$ & $141.0(139.0,143.0)$ & $140.0(139.0,142.0)$ & 0.1949 \\
\hline$<135$ & $2(2)$ & $0(0)$ & $2(3)$ & 0.7669 \\
\hline $135-145$ & $97(96)$ & $39(98)$ & $58(95)$ & \\
\hline$>145$ & $2(2)$ & $1(3)$ & $1(2)$ & \\
\hline $\mathrm{K}(\mathrm{mmol} / \mathrm{L})$ & $4.1(3.7,4.5)$ & $4.1(3.6,4.6)$ & $4.1(3.7,4.4)$ & 0.5923 \\
\hline$<3.5$ & $15(15)$ & $4(10)$ & $11(18)$ & 0.5247 \\
\hline $3.5-5.5$ & $81(80)$ & $34(85)$ & 47 (77) & \\
\hline$>5.5$ & $5(5)$ & $2(5)$ & $3(5)$ & \\
\hline White blood cell count $\left(\times 10^{9} / \mathrm{L}\right)$ & $6.0(4.5,8.7)$ & $6.1(5.0,9.7)$ & $5.8(4.4,8.2)$ & 0.5289 \\
\hline $4-10, n(\%)$ & $66 / 100(66)$ & $26(65)$ & $40 / 60(67)$ & 0.9049 \\
\hline$<4, n(\%)$ & $16 / 100(16)$ & $6(15)$ & $10 / 60(17)$ & \\
\hline$>10, n(\%)$ & $18 / 100(18)$ & $8(20)$ & $10 / 60(17)$ & \\
\hline Lymphocyte count $\left(\times 10^{9} / \mathrm{L}\right)$ & $0.8(0.6,1.3)$ & $0.8(0.6,1.3)$ & $0.8(0.6,1.2)$ & 0.8301 \\
\hline$<0.8, n(\%)$ & $51 / 100(51)$ & $22(55)$ & $29 / 60(48)$ & 0.5135 \\
\hline Hemoglobin $(g / L)$ & $121.0(110.0,135.5)$ & $117.5(108.5,134.0)$ & $123.0(113.0,136.5)$ & 0.2601 \\
\hline Platelet count $\left(\times 10^{9} / \mathrm{L}\right)$ & $202.5(150.0,261.5)$ & $189.0(145.5,251.5)$ & $206.0(162.0,269.5)$ & 0.5129 \\
\hline$<100, n(\%)$ & $3 / 100(3)$ & $1(3)$ & $2 / 60(3)$ & 1.0000 \\
\hline Alanine transaminase $(\mathrm{U} / \mathrm{L})$ & $32.0(21.0,54.0)$ & $32.0(23.0,54.0)$ & $34.0(20.0,53.0)$ & 0.6342 \\
\hline$\leqslant 40, n(\%)$ & $62(61)$ & $25(63)$ & $37(61)$ & 0.8523 \\
\hline$>40, n(\%)$ & $39(39)$ & $15(38)$ & $24(39)$ & \\
\hline Creatinine $>133(\mu \mathrm{mol} / \mathrm{L}), n(\%)$ & $6(6)$ & $2(5)$ & $4(7)$ & 1.0000 \\
\hline
\end{tabular}




\begin{tabular}{|c|c|c|c|c|}
\hline & & & & (Continued) \\
\hline Characteristics & $\begin{array}{l}\text { Total } \\
n=101\end{array}$ & $\begin{array}{l}\text { ACEI/ARB } \\
n=40\end{array}$ & $\begin{array}{l}\text { Non-ACEI/ARB } \\
n=61\end{array}$ & $P$ value \\
\hline Lactate dehydrogenase (U/L) & $336.0(238.0,455.0)$ & $307.0(230.0,403.0)$ & $357.0(248.0,481.5)$ & 0.0828 \\
\hline$\leqslant 245, n(\%)$ & $28 / 100(28)$ & $13(33)$ & $15 / 60(25)$ & 0.4132 \\
\hline$>245, n(\%)$ & $72 / 100(72)$ & $27(68)$ & $45 / 60(75)$ & \\
\hline Creatine kinase $(\mathrm{U} / \mathrm{L})$ & $15.0(12.0,36.0)$ & $15.0(11.0,23.5)$ & $17.0(13.0,43.0)$ & 0.2639 \\
\hline$\leqslant 185, n(\%)$ & $95 / 99(96)$ & $38(95)$ & $57 / 59(97)$ & 1.0000 \\
\hline$>185, n(\%)$ & 4/99 (4) & $2(5)$ & $2 / 59(3)$ & \\
\hline Brain natriuretic peptide $(\mathrm{pg} / \mathrm{mL})$ & $58.6(29.9,110.6)$ & $55.7(28.9,119.7)$ & $61.2(32.6,108.6)$ & 0.6878 \\
\hline$\geqslant 100, n(\%)$ & $17 / 62(27)$ & $7 / 25(28)$ & $10 / 37(27)$ & 0.9329 \\
\hline Cardiac troponin I $(\mathrm{pg} / \mathrm{mL})$ & $7.2(3.1,19.9)$ & $5.7(2.5,20.8)$ & $9.1(4.1,17.2)$ & 0.2629 \\
\hline$>28, n(\%)$ & 18/99 (18) & $7(18)$ & $11 / 59(19)$ & 0.8848 \\
\hline Prothrombin time (s) & $11.4(10.4,12.4)$ & $11.5(10.2,12.6)$ & $11.3(10.5,12.3)$ & 0.9857 \\
\hline$<16, n(\%)$ & 97/99 (98) & $38 / 39$ (97) & $59 / 60(98)$ & 1.0000 \\
\hline$\geqslant 16, n(\%)$ & 2/99 (2) & $1 / 39(3)$ & $1 / 60(2)$ & \\
\hline D-dimer $(\mu \mathrm{g} / \mathrm{mL})$ & $1.3(0.6,4.5)$ & $1.2(0.5,2.7)$ & $1.4(0.7,5.5)$ & 0.1759 \\
\hline$\leqslant 1, n(\%)$ & $41 / 99(41)$ & $16 / 39(41)$ & $25 / 60(42)$ & 0.9496 \\
\hline$>1, n(\%)$ & $58 / 99(59)$ & $23 / 39(59)$ & $35 / 60(58)$ & \\
\hline Serum ferritin $(\mu \mathrm{g} / \mathrm{L})$ & $576.9(334.5,980.4)$ & $577.1(334.5,938.8)$ & $548.8(336.6,1001.0)$ & 0.9853 \\
\hline$\leqslant 300, n(\%)$ & $17 / 83(20)$ & $8 / 35(23)$ & 9/48 (19) & 0.6471 \\
\hline$>300, n(\%)$ & $66 / 83(80)$ & $27 / 35(77)$ & $39 / 48(81)$ & \\
\hline Procalcitonin $(\mathrm{ng} / \mathrm{mL})$ & $0.1(0.1,0.2)$ & $0.1(0.1,0.1)$ & $0.1(0.1,0.2)$ & 0.2729 \\
\hline$<0.1, n(\%)$ & $36 / 65(55)$ & $16 / 24(67)$ & 20/41 (49) & 0.4237 \\
\hline$\geqslant 0.1$ to $<0.25, n(\%)$ & $21 / 65(32)$ & $5 / 24(21)$ & $16 / 41(39)$ & \\
\hline$\geqslant 0.25$ to $<0.5, n(\%)$ & $2 / 65(3)$ & $1 / 24(4)$ & $1 / 41(2)$ & \\
\hline$\geqslant 0.5, n(\%)$ & $6 / 65(9)$ & $2 / 24(8)$ & $4 / 41(10)$ & \\
\hline Interleukin $6(\mathrm{pg} / \mathrm{mL})$ & $7.5(6.0,10.9)$ & $7.0(5.7,9.8)$ & $8.0(6.1,11.4)$ & 0.1429 \\
\hline \multicolumn{5}{|l|}{ Imaging features } \\
\hline Consolidation, $n(\%)$ & $64(63)$ & $24(60)$ & $40(66)$ & 0.5696 \\
\hline Ground-glass opacity, $n(\%)$ & $82(81)$ & $32(80)$ & $50(82)$ & 0.8046 \\
\hline $\begin{array}{l}\text { Bilateral pulmonary infiltration, } \\
n(\%)\end{array}$ & $82(81)$ & $33(83)$ & $49(80)$ & 0.7847 \\
\hline \multicolumn{5}{|l|}{ Disease severity scores } \\
\hline SOFA & $3.5(2.0,4.0)$ & $2.0(1.0,4.0)$ & $4.0(2.0,4.5)$ & 0.2917 \\
\hline CURB-65 & $1.0(1.0,2.0)$ & $1.0(1.0,2.0)$ & $1.0(0.0,2.0)$ & 0.6631 \\
\hline $0-1, n(\%)$ & $73(72)$ & $28(70)$ & $45(74)$ & 0.7230 \\
\hline $2, n(\%)$ & $20(20)$ & $9(23)$ & $11(18)$ & \\
\hline $3-5, n(\%)$ & $8(8)$ & $3(8)$ & $5(8)$ & \\
\hline
\end{tabular}

Data were presented as median (IQR) or number (proportion). $P$ values for comparison between ACEI/ARB group and non-ACEI/ARB group were calculated by Mann-Whitney U test, $\chi^{2}$ test or Fisher's exact test where appropriate. Abbreviation: COPD, chronic obstructive pulmonary disease; SOFA, sequential organ failure assessment.

during hospitalization. Hospital length of stay and duration of viral shedding for discharged patients were similar between ACEI/ARB and non-ACEI/ARB groups (Table 4). We did not include the non-survivors in the analysis for viral-shedding duration because it has been previously reported that non-survivors had prolonged viral shedding until death [6].

Further multivariable analysis adjusted for age and gender did not provide statistical evidence for association between ACEI/ARB treatment and severe clinical outcome in COVID-19 patients (Table 5). As previously reported in the total population [6], older age, increased D-dimer values $(>1 \mu \mathrm{g} / \mathrm{mL})$ and higher qSOFA scores were associated with higher risks of in-hospital mortality in hypertensive COVID-19 patients in the Logistic model (Table 6).

\section{Discussion}

The hypothesis that ACEI or ARB treatment changes COVID-19 disease progression was based in part on potential impact of RAS antagonists on ACE2 expression. Theoretically, does administration of an ACEI or ARB 
Table 2 Anti-hypertensive treatment therapies

\begin{tabular}{|c|c|c|c|c|}
\hline Treatment & $\begin{array}{l}\text { Total } \\
n=101\end{array}$ & $\begin{array}{l}\mathrm{ACEI} / \mathrm{ARB} \\
n=40\end{array}$ & $\begin{array}{l}\text { Non-ACEI/ARB } \\
n=61\end{array}$ & $P$ value \\
\hline$\overline{\mathrm{ACEI}}$ & $8(8)$ & $8(20)$ & 0 & 0.0011 \\
\hline ARB & $33(33)$ & $33(83)$ & 0 & $<0.0001$ \\
\hline$\alpha$-blocker & $2(20)$ & $1(3)$ & $1(2)$ & 1.0000 \\
\hline$\beta$-blocker & $15(15)$ & $5(13)$ & $10(16)$ & 0.5905 \\
\hline $\mathrm{CCB}$ & 77 (77) & $19(48)$ & $58(95)$ & $<0.0001$ \\
\hline Diuretics & $9(9)$ & $6(15)$ & $3(5)$ & 0.1669 \\
\hline
\end{tabular}

Data were presented as number (proportion). $P$ values for comparison between ACEI/ARB group and non-ACEI/ARB group were calculated by $\chi^{2}$ test or Fisher's exact test where appropriate. Abbreviation: ACEI, angiotensin-converting enzyme inhibitor; ARB, angiotensin II receptor blocker; CCB, calcium channel blocker.

have an impact on ACE2 expression or activity? First of all, it has been shown that ACEIs do not affect ACE2 activity [15]. In terms of ACE2 expression, olmesartan, but not other ACEIs, ARBs or calcium channel blockers, has been found to increase urinary ACE2 levels in hypertensive patients [37,38]. Also, ACEI treatment has been reported to increase serum ACE2 activity in diabetic patients [39]. Yet the soluble ACE2 (sACE2) levels do not always reflect the tissue ACE2 expression in lung or other organs. In fact, it is reasonable to hypothesize that sACE2 may even negatively correlate with tissue ACE2 since sACE2 is cleaved from the membrane-bound enzyme before being released into the blood stream. Also, it has to be noted that increase in ACE2 enzymatic activity does not always match the upregulation of ACE2 expression

Table 3 Anti-hypertensive treatment therapies by combination

\begin{tabular}{ll}
\hline Treatment & Number \\
\hline $\mathrm{A}^{\mathrm{a}}$ & 14 \\
$\mathrm{~B}$ & 3 \\
$\mathrm{C}$ & 48 \\
$\mathrm{~A}+\mathrm{B}$ & 3 \\
$\mathrm{~A}+\mathrm{C}$ & 17 \\
$\mathrm{~A}+\mathrm{D}$ & 2 \\
$\mathrm{~B}+\mathrm{C}$ & 7 \\
$\mathrm{C}+\mathrm{D}$ & 3 \\
$\mathrm{~A}+\mathrm{C}+\mathrm{D}$ & 2 \\
A + B + C + D & 2 \\
Total & 101
\end{tabular}

Anti-hypertensive treatment as either mono-therapy or combined therapies. ${ }^{a}$ One of 14 patients took valsartan and benazepril. All the other 13 patients were on either ACEI or ARB as mono-therapy. A means angiotensinconverting enzyme inhibitor (ACEI) or angiotensin II receptor blocker (ARB); B means $\beta$-blocker; $\mathrm{C}$ means calcium channel blocker (CCB); D means diuretics. ACEI refers to benazepril in this cohort. ARB medications include valsartan, irbesartan, telmisartan, and candesartan. $\beta$-blockers used in this cohort include metoprolol and bisoprolol. CCBs include amlodipine, nifedipine, felodipine, lacidipine, and lercanidipine. Diuretics include hydrochlorothiazide and spironolactone. Other uncommon medications not listed in this table were terazosin ( $\alpha$-blocker) and traditional Chinese medicine with un-specified formula.
[40,41]. Therefore, change in pulmonary tissue ACE2 after ACEI or ARB treatment has to be directly studied to provide answers for this question, although studies of upper respiratory tract ACE2 expression or ACE2 enzyme activity in bronchoalveolar lavage fluid might provide indirect evidence $[42,43]$.

Hypertensive animal models might be a surrogate before clinical evidence is available. In rat cardiac myocytes, Ang II significantly reduces ACE2 activity and downregulates ACE2 mRNA expression; these effects can be blocked by the ARB losartan, indicating that Ang II regulates ACE2 [24]. Mice deficient for ACE show markedly improved disease in an ARDS model induced by acid aspiration or sepsis, and recombinant ACE2 can protect mice from severe acute lung injury [44]. In mice and rat models, ARB treatment consistently increases ACE2 mRNA and protein levels in heart, kidney, and aorta tissue, although its effect on lung ACE2 expression has not been studied. Also, the effects of ACEI administration on ACE2 expression differs among experiment models and tissues [45]. Animal models of SARS-CoV-2 infection that recapitulate the pathogenesis of severe COVID-19 are needed to better understand the effects of ACE inhibitors and ARBs.

In this single-center retrospective study, we did not observe difference in clinical presentations of patients with or without ACEI/ARB treatment on admission. Nor did we find any important differences in severe clinical outcomes, namely in-hospital death, ICU admission, and mechanical ventilation, in these two groups. However, our preliminary analysis was limited by the retrospective nature of the cohort, its small sample size, and possibly nonrecorded data as the prescription records for drugs not directly relevant to COVID-19 treatment may be incomplete. Only patients with complete anti-hypertensive treatment information were included in the analysis, but this strategy, by excluding about half the hypertensive cases in the original hypertensive cohort, reduced statistical power of this study. Based on the percentages of in-hospital mortality and ICU admission reported in this cohort, around 500-1000 patients for each group would be necessary to provide 
Table 4 Treatments and clinical outcomes

\begin{tabular}{|c|c|c|c|c|}
\hline & $\begin{array}{l}\text { Total } \\
n=101\end{array}$ & $\begin{array}{l}\text { ACEI/ARB } \\
n=40\end{array}$ & $\begin{array}{l}\text { Non-ACEI/ARB } \\
n=61\end{array}$ & $P$ value \\
\hline \multicolumn{5}{|l|}{ Treatments } \\
\hline Antibiotic drugs, $n(\%)$ & $95(94)$ & $36(90)$ & $59(97)$ & 0.3334 \\
\hline Antiviral drugs $\mathrm{s}^{\mathrm{a}}, n(\%)$ & $44(44)$ & $17(43)$ & $27(44)$ & 0.8613 \\
\hline Lopinavir-ritonavir, $n(\%)$ & $16(16)$ & $6(15)$ & $10(16)$ & 0.8512 \\
\hline Corticosteroids, $n(\%)$ & $28(28)$ & $11(28)$ & $17(28)$ & 0.9677 \\
\hline Intravenous immunoglobin, $n(\%)$ & $36(36)$ & $15(38)$ & $21(34)$ & 0.7524 \\
\hline \multicolumn{5}{|l|}{ Highest oxygenation support therapy } \\
\hline No oxygenation support, $n(\%)$ & $6(6)$ & $5(12)$ & $1(2)$ & 0.1087 \\
\hline $\mathrm{NC}, n(\%)$ & $56(55)$ & $23(58)$ & $33(54)$ & \\
\hline HFNC, $n(\%)$ & $16(16)$ & $5(12)$ & $11(18)$ & \\
\hline NIMV, $n(\%)$ & $4(4)$ & $0(0)$ & $4(6)$ & \\
\hline $\mathrm{IMV}, n(\%)$ & $19(19)$ & $7(18)$ & $12(20)$ & \\
\hline Renal replacement therapy, $n(\%)$ & $8(8)$ & $3(8)$ & $5(8)$ & 1.0000 \\
\hline \multicolumn{5}{|l|}{ Complications } \\
\hline Respiratory failure, $n(\%)$ & $53(52)$ & $17(43)$ & $36(59)$ & 0.1040 \\
\hline Sepsis, $n(\%)$ & $55(54)$ & $19(48)$ & $36(59)$ & 0.2557 \\
\hline Sepsis shock, $n(\%)$ & $13(13)$ & $5(13)$ & $8(13)$ & 0.9281 \\
\hline ARDS, $n(\%)$ & $33(33)$ & $11(28)$ & $22(36)$ & 0.3694 \\
\hline Acute kidney injury, $n(\%)$ & $11(11)$ & $5(13)$ & $6(10)$ & 0.9253 \\
\hline Acute cardiac injury, $n(\%)$ & $24(24)$ & $9(23)$ & $15(25)$ & 0.8093 \\
\hline Acidosis, $n(\%)$ & $9(9)$ & $3(8)$ & $6(10)$ & 0.9633 \\
\hline Secondary infection, $n(\%)$ & $9(9)$ & $4(10)$ & $5(8)$ & 1.0000 \\
\hline Hypoproteinemia, $n(\%)$ & $21(21)$ & $10(25)$ & $11(18)$ & 0.3988 \\
\hline Coagulopathy, $n(\%)$ & $15(15)$ & $7(18)$ & $8(13)$ & 0.5444 \\
\hline \multicolumn{5}{|l|}{ Outcomes } \\
\hline ICU admission, $n(\%)$ & $25(25)$ & $8(20)$ & $17(28)$ & 0.3702 \\
\hline Death, $n(\%)$ & $32(32)$ & $11(28)$ & $21(34)$ & 0.4643 \\
\hline Mechanical ventilation ${ }^{\mathrm{b}}, n(\%)$ & $23(23)$ & $7(18)$ & $16(26)$ & 0.3062 \\
\hline Hospital length of stay ${ }^{\mathrm{c}}$ (day) & $12.0(8.5,15.0)$ & $13.0(9.0,15.0)$ & $12.0(7.0,14.5)$ & 0.1955 \\
\hline $\begin{array}{l}\text { Duration of viral shedding after illness } \\
\text { onset }^{\mathrm{c}} \text { (day) }\end{array}$ & $18.0(15.0,23.0)$ & $19.0(15.0,23.0)$ & $18.0(14.5,23.0)$ & 0.8123 \\
\hline
\end{tabular}

Data were presented as median (IQR) or number (proportion). $P$ values for comparison between ACEI/ARB group and non-ACEI/ARB group were calculated by Mann-Whitney U test, $\chi^{2}$ test or Fisher's exact test where appropriate. ${ }^{a}$ Antiviral treatment includes oseltamivir $(n=3)$, ganciclovir $(n=4)$, lopinavirritonavir $(n=16)$, arbidol $(n=23)$, ribavirin $(n=5)$, interferon $\alpha(n=3)$, and remdesivir $(n=1)$. ${ }^{\mathrm{b}}$ Mechanical ventilation is the combination of IMV and NIMV. ${ }^{\mathrm{c}}$ Only for 69 patients who survived and were discharged. Abbreviations: NC, nasal canula; HFNC, high-flow nasal cannula; NIMV, non-invasive mechanical ventilation; IMV, invasive mechanical ventilation; ARDS, acute respiratory distress syndrome; ICU, intensive care unit.

Table 5 Risk of ACEI/ARB administration on severe COVID-19 outcome

\begin{tabular}{|c|c|c|c|c|c|c|}
\hline & \multicolumn{2}{|c|}{ Death } & \multicolumn{2}{|c|}{ ICU admission } & \multicolumn{2}{|c|}{ IMV } \\
\hline & OR $(95 \%$ CI $)$ & $P$ value & OR $(95 \% \mathrm{CI})$ & $P$ value & OR $(95 \% \mathrm{CI})$ & $P$ value \\
\hline ACEI/ARB & $0.73(0.29-1.82)$ & 0.4994 & $0.65(0.25-1.70)$ & 0.3798 & $0.87(0.31-2.43)$ & 0.7860 \\
\hline $\begin{array}{r}\text { ACEI/ARB } \\
(\text { adjusted) }\end{array}$ & $0.78(0.32-1.93)$ & 0.5894 & $0.68(0.26-1.81)$ & 0.4431 & $0.92(0.32-2.63)$ & 0.8796 \\
\hline
\end{tabular}

The odds ratio (OR) and 95\% confidence interval (CI) for treatment with ACEI/ARB vs. non-ACEI/ARB on severe outcomes of COVID-19 were estimated by Logistic models. ${ }^{a}$ Adjusted for age and gender. Abbreviations: ICU, intensive care unit; IMV, invasive mechanical ventilation.

statistical difference given two-tailed $\alpha=0.05$ and $\beta=0.2$. Although this cohort had enough power to detect the association between older age and increased COVID-19 mortality (Table 6), it is under-powered for the marginal effect of ACEI/ARB on altered clinical outcome if there was association.

Other confounding factors including age should be carefully evaluated before drawing the final conclusion 
Table 6 Risk factors associated with in-hospital death in hypertensive patients on medication

\begin{tabular}{llll}
\hline & Univariable OR (95\% CI) & $P$ value & $P$ value \\
\hline Age (year) & $1.07(1.03-1.12)$ & 0.0025 & $1.04(0.99-1.10)$ \\
Diabetes & $1.76(0.63-4.91)$ & 0.2820 & 0.1059 \\
qSOFA score & $4.77(2.07-10.96)$ & 0.00024 & $5.41(2.18-13.48)$ \\
D-dimer $>1 \mu \mathrm{g} / \mathrm{mL}$ & $3.43(1.30-9.02)$ & 0.0125 & $3.64(1.10-11.99)$ \\
\hline
\end{tabular}

Abbreviation: OR, odds ratio; qSOFA, quick sequential organ failure assessment.

from retrospective analysis. It has to be noted that while ACEI/ARB are the most popular anti-hypertensive drug in the US [46], CCBs are more widely used in China [7,47]. This partially explains the high CCB usage in the nonACEI/ARB group (Tables 2 and 3). After adjustment for the difference of CCB prescription, ACEI/ARB still does not show association with COVID-19 mortality (OR 0.70, 95\% CI $0.24-1.99, P=0.5011)$. We did not observe association between CCB usage and COVID-19 mortality, either, from this cohort (data not shown). Pre-existing heart failure should also be assessed because RAS antagonists including ACEI, ARB, and aldosterone inhibitors were basic treatment options for chronic heart failure. Proper multivariable adjustment on confounding factors should be implemented in retrospective analysis. We did not adjust for history of heart failure concerning the small sample size and the self-reporting nature of this factor. We did not have information for viral load of patients on admission, because only qualitative RT-PCR test results were available in the medical records. It would be interesting to examine whether chronic ACEI or ARB treatment would affect virus replication in future studies.

Similar to our findings, no association was observed between ACEI/ARB prescription and poor clinical prognosis or severe disease of COVID-19 in several other retrospective cohorts in China [27,28,48,49] or globally [29-32]. Compared with these reports, patients in this cohort were sicker on admission and the in-hospital mortality was higher (32\%). This likely related to the study hospital being a referral center with severe cases during early epidemic in Wuhan.

With accumulating information from multiple large cohort studies, it seems clear that prior receipt of ACEI or ARB does not bring additional risks of hospital admission or poor prognosis of COVID-19. The discussion has therefore been gradually shifted to whether chronic treatment of ACEI or ARB before COVID-19 onset is associated with less severe illness. ACEI and ARB have been reported with protective effect in a previous metaanalysis for pneumonia-related mortality in general [10]. In terms of COVID-19, de Abajo et al. found a decreased risk of COVID-19 hospital admission in the subgroup analysis of diabetic patients with RAS inhibitors [31]. Information from other large COVID-19 case series and preferably population-based cohort studies, optimally linked to serologic testing, are necessary to determine whether receipt of ACEI or ARBs are associated with reduced risk of symptomatic SARS-CoV-2 infection and especially of severe COVID-19 illness. Systematic analysis combining data from multiple studies would be helpful in answering this question. There have also been randomized clinical trials in progress studying the efficacy of acute initiation of ACEI for treatment of COVID-19 (e.g., NCT04366050 and NCT04355429).

In conclusion, there has been accumulating evidence for no association between chronic ACEI/ARB treatment and severe outcome of COVID-19. Meanwhile, current studies are pointing toward a putative protective role of prior ACEI or ARB receipt in COVID-19 illness, which awaits further evidence for confirmation. Several professional societies have released statements on continuing current anti-hypertensive treatment during the COVID-19 pandemic [50], considering the risk of destabilizing blood pressure after changing medications. We agree that the best option is to continue previous therapy and wait for further evidence.

\section{Acknowledgements}

We acknowledge all health-care workers involved in the diagnosis and treatment of patients in Jinyintan Hospital in Wuhan, China. This work was supported by Major Projects of National Science and Technology on New Drug Creation and Development (Nos. 2020ZX09201001 and 2020ZX09201012); Chinese Academy of Medical Sciences (CAMS) Emergency Project of COVID-19 (No. 2020HY320001).

\section{Compliance with ethics guidelines}

Dr. Frederick G. Hayden declares he has served as non-compensated consultant to Cidara, GlaxoSmithKline, Gilead Sciences, resTORbio, Regeneron, SAB Biotherapeutics on coronavirus interventions, outside the submitted work. All other authors declare no competing interests. The study was performed in accordance with the Helsinki Declaration of 1975, and was approved by the Research Ethics Commission of Jinyintan Hospital (KY-2020-01.01). Informed consent was waived by the Research Ethics Commission. 


\section{References}

1. World Health Organization. Coronavirus disease (COVID-19) outbreak situation. 2020. https://www.who.int/emergencies/diseases/novel-coronavirus-2019 (accessed May 24, 2020)

2. Guan WJ, Ni ZY, Hu Y, Liang WH, Ou CQ, He JX, Liu L, Shan H, Lei CL, Hui DSC, Du B, Li LJ, Zeng G, Yuen KY, Chen RC, Tang CL, Wang T, Chen PY, Xiang J, Li SY, Wang JL, Liang ZJ, Peng YX, Wei L, Liu Y, Hu YH, Peng P, Wang JM, Liu JY, Chen Z, Li G, Zheng ZJ, Qiu SQ, Luo J, Ye CJ, Zhu SY, Zhong NS; China Medical Treatment Expert Group for COVID-19. Clinical characteristics of coronavirus disease 2019 in China. N Engl J Med 2020; 382(18): 1708-1720

3. Wang D, Hu B, Hu C, Zhu F, Liu X, Zhang J, Wang B, Xiang H, Cheng Z, Xiong Y, Zhao Y, Li Y, Wang X, Peng Z. Clinical characteristics of 138 hospitalized patients with 2019 novel coronavirus-infected pneumonia in Wuhan, China. JAMA 2020; 323(11): 1061

4. Guan WJ, Liang WH, Zhao Y, Liang HR, Chen ZS, Li YM, Liu XQ, Chen RC, Tang CL, Wang T, Ou CQ, Li L, Chen PY, Sang L, Wang W, Li JF, Li CC, Ou LM, Cheng B, Xiong S, Ni ZY, Xiang J, Hu Y, Liu L, Shan H, Lei CL, Peng YX, Wei L, Liu Y, Hu YH, Peng P, Wang JM, Liu JY, Chen Z, Li G, Zheng ZJ, Qiu SQ, Luo J, Ye CJ, Zhu SY, Cheng LL, Ye F, Li SY, Zheng JP, Zhang NF, Zhong NS, He JX; China Medical Treatment Expert Group for COVID-19. Comorbidity and its impact on 1590 patients with COVID-19 in China: a nationwide analysis. Eur Respir J 2020; 55(5): 2000547

5. Chen T, Wu D, Chen H, Yan W, Yang D, Chen G, Ma K, Xu D, Yu H, Wang H, Wang T, Guo W, Chen J, Ding C, Zhang X, Huang J, Han M, Li S, Luo X, Zhao J, Ning Q. Clinical characteristics of 113 deceased patients with coronavirus disease 2019: retrospective study. BMJ 2020; 368: m1091

6. Zhou F, Yu T, Du R, Fan G, Liu Y, Liu Z, Xiang J, Wang Y, Song B, Gu X, Guan L, Wei Y, Li H, Wu X, Xu J, Tu S, Zhang Y, Chen H, Cao B. Clinical course and risk factors for mortality of adult inpatients with COVID-19 in Wuhan, China: a retrospective cohort study. Lancet 2020; 395(10229): 1054-1062

7. Lu J, Lu Y, Wang X, Li X, Linderman GC, Wu C, Cheng X, Mu L, Zhang H, Liu J, Su M, Zhao H, Spatz ES, Spertus JA, Masoudi FA, Krumholz HM, Jiang L. Prevalence, awareness, treatment, and control of hypertension in China: data from 1.7 million adults in a population-based screening study (China PEACE Million Persons Project). Lancet 2017; 390(10112): 2549-2558

8. Fang L, Karakiulakis G, Roth M. Are patients with hypertension and diabetes mellitus at increased risk for COVID-19 infection? Lancet Respir Med 2020; 8(4): e21

9. Liao Y, Cheng X, Zeng Q, Chen Z, Wang Z, Yuan J, Wang X, Zhou Z, Wei Y, Cao G. Expert recommendations for management and treatment of cardiovascular diseases under the epidemic situation of novel coronavirus pneumonia in Hubei Province. J Clin Cardiol (Lin Chuang Xin Xue Guan Bing Za Zhi) 2020; 36(3): 201-203 (in Chinese)

10. Caldeira D, Alarcao J, Vaz-Carneiro A, Costa J. Risk of pneumonia associated with use of angiotensin converting enzyme inhibitors and angiotensin receptor blockers: systematic review and meta-analysis. BMJ, 2012; 345: e4260

11. Mortensen EM, Nakashima B, Cornell J, Copeland LA, Pugh MJ,
Anzueto A, Good C, Restrepo MI, Downs JR, Frei CR, Fine MJ. Population-based study of statins, angiotensin II receptor blockers, and angiotensin-converting enzyme inhibitors on pneumonia-related outcomes. Clin Infect Dis 2012; 55(11): 1466-1473

12. Kuba K, Imai Y, Rao S, Gao H, Guo F, Guan B, Huan Y, Yang P, Zhang Y, Deng W, Bao L, Zhang B, Liu G, Wang Z, Chappell M, Liu Y, Zheng D, Leibbrandt A, Wada T, Slutsky AS, Liu D, Qin C, Jiang C, Penninger JM. A crucial role of angiotensin converting enzyme 2 (ACE2) in SARS coronavirus-induced lung injury. Nat Med 2005; 11(8): 875-879

13. Yan Y, Liu Q, Li N, Du J, Li X, Li C, Jin N, Jiang C. Angiotensin II receptor blocker as a novel therapy in acute lung injury induced by avian influenza A H5N1 virus infection in mouse. Sci China Life Sci 2015; 58(2): 208-211

14. Donoghue M, Hsieh F, Baronas E, Godbout K, Gosselin M, Stagliano N, Donovan M, Woolf B, Robison K, Jeyaseelan R, Breitbart RE, Acton S. A novel angiotensin-converting enzymerelated carboxypeptidase (ACE2) converts angiotensin I to angiotensin 1-9. Circ Res 2000; 87(5): E1-E9

15. Tipnis SR, Hooper NM, Hyde R, Karran E, Christie G, Turner AJ. A human homolog of angiotensin-converting enzyme. Cloning and functional expression as a captopril-insensitive carboxypeptidase. J Biol Chem 2000; 275(43): 33238-33243

16. Santos RA, Simoes e Silva AC, Maric C, Silva DM, Machado RP, de Buhr I, Heringer-Walther S, Pinheiro SV, Lopes MT, Bader M, Mendes EP, Lemos VS, Campagnole-Santos MJ, Schultheiss HP, Speth R, Walther T. Angiotensin-(1-7) is an endogenous ligand for the G protein-coupled receptor Mas. Proc Natl Acad Sci USA 2003; 100(14): 8258-8263

17. Wösten-van Asperen RM, Lutter R, Specht PA, Moll GN, van Woensel JB, van der Loos CM, van Goor H, Kamilic J, Florquin S, Bos AP. Acute respiratory distress syndrome leads to reduced ratio of ACE/ACE2 activities and is prevented by angiotensin-(1-7) or an angiotensin II receptor antagonist. J Pathol 2011; 225(4): 618-627

18. Li W, Moore MJ, Vasilieva N, Sui J, Wong SK, Berne MA, Somasundaran M, Sullivan JL, Luzuriaga K, Greenough TC, Choe $\mathrm{H}$, Farzan M. Angiotensin-converting enzyme 2 is a functional receptor for the SARS coronavirus. Nature 2003; 426(6965): 450454

19. Letko M, Marzi A, Munster V. Functional assessment of cell entry and receptor usage for SARS-CoV-2 and other lineage B betacoronaviruses. Nat Microbiol 2020; 5(4): 562-569

20. Zhou P, Yang XL, Wang XG, Hu B, Zhang L, Zhang W, Si HR, Zhu Y, Li B, Huang CL, Chen HD, Chen J, Luo Y, Guo H, Jiang RD, Liu MQ, Chen Y, Shen XR, Wang X, Zheng XS, Zhao K, Chen QJ, Deng F, Liu LL, Yan B, Zhan FX, Wang YY, Xiao GF, Shi ZL. A pneumonia outbreak associated with a new coronavirus of probable bat origin. Nature 2020; 579(7798): 270-273

21. Yan R, Zhang Y, Li Y, Xia L, Guo Y, Zhou Q. Structural basis for the recognition of SARS-CoV-2 by full-length human ACE2. Science 2020; 367(6485): 1444-1448

22. Hamming I, Timens W, Bulthuis ML, Lely AT, Navis G, van Goor $\mathrm{H}$. Tissue distribution of ACE2 protein, the functional receptor for SARS coronavirus. A first step in understanding SARS pathogenesis. J Pathol 2004; 203(2): 631-637

23. Zhao Y, Zhao Z, Wang Y, Zhou Y, Ma Y, Zuo W. Single-cell RNA expression profiling of ACE2, the receptor of SARS-CoV-2. bioRxiv 2020; doi: 10.1101/2020.01.26.919985 
24. Gallagher PE, Ferrario CM, Tallant EA. Regulation of ACE2 in cardiac myocytes and fibroblasts. Am J Physiol Heart Circ Physiol 2008; 295(6): H2373-H2379

25. Burrell LM, Risvanis J, Kubota E, Dean RG, MacDonald PS, Lu S, Tikellis C, Grant SL, Lew RA, Smith AI, Cooper ME, Johnston CI. Myocardial infarction increases ACE2 expression in rat and humans. Eur Heart J 2005; 26(4): 369-375, discussion 322-364

26. Danser AHJ, Epstein M, Batlle D. Renin-angiotensin system blockers and the COVID-19 pandemic: at present there is no evidence to abandon renin-angiotensin system blockers. Hypertension 2020; 75(6): 1382-1385

27. Huang Z, Cao J, Yao Y, Jin X, Luo Z, Xue Y, Zhu C, Song Y, Wang Y, Zou Y, Qian J, Yu K, Gong H, Ge J. The effect of RAS blockers on the clinical characteristics of COVID-19 patients with hypertension. Ann Transl Med 2020; 8(7): 430

28. Zhang P, Zhu L, Cai J, Lei F, Qin JJ, Xie J, Liu YM, Zhao YC, Huang X, Lin L, Xia M, Chen MM, Cheng X, Zhang X, Guo D, Peng Y, Ji YX, Chen J, She ZG, Wang Y, Xu Q, Tan R, Wang H, Lin J, Luo P, Fu S, Cai H, Ye P, Xiao B, Mao W, Liu L, Yan Y, Liu M, Chen M, Zhang XJ, Wang X, Touyz RM, Xia J, Zhang BH, Huang X, Yuan Y, Rohit L, Liu PP, Li H. Association of inpatient use of angiotensin converting enzyme inhibitors and angiotensin II receptor blockers with mortality among patients with hypertension hospitalized with COVID-19. Circ Res 2020; [Epub ahead of print] doi: 10.1161/CIRCRESAHA.120.317134

29. Mehta N, Kalra A, Nowacki AS, Anjewierden S, Han Z, Bhat P, Carmona-Rubio AE, Jacob $M$, Procop $G$ W, Harrington $S$, Milinovich A, Svensson LG, Jehi L, Young JB, Chung MK. Association of use of angiotensin-converting enzyme inhibitors and angiotensin II receptor blockers with testing positive for coronavirus disease 2019 (COVID-19). JAMA Cardiol 2020; [Epub ahead of print] doi: 10.1001/jamacardio.2020.1855

30. Reynolds HR, Adhikari S, Pulgarin C, Troxel AB, Iturrate E, Johnson SB, Hausvater A, Newman JD, Berger JS, Bangalore S, Katz SD, Fishman GI, Kunichoff D, Chen Y, Ogedegbe G, Hochman JS. Renin-angiotensin-aldosterone system inhibitors and risk of COVID-19. N Engl J Med 2020; [Epub ahead of print] doi: 10.1056/NEJMoa2008975

31. de Abajo FJ, Rodríguez-Martín S, Lerma V, Mejía-Abril G, Aguilar M, García-Luque A, Laredo L, Laosa O, Centeno-Soto GA, Ángeles Gálvez M, Puerro M, González-Rojano E, Pedraza L, de Pablo I, Abad-Santos F, Rodríguez-Mañas L, Gil M, Tobías A, RodríguezMiguel A, Rodríguez-Puyol D, Barreira-Hernandez D, Zubiaur P, Santos-Molina E, Pintos-Sánchez E, Navares-Gómez M, Aparicio RM, García-Rosado V, Gutiérrez-Ortega C, Pérez C, Ascaso A, Elvira C. Use of renin-angiotensin-aldosterone system inhibitors and risk of COVID-19 requiring admission to hospital: a casepopulation study. Lancet 2020; 395(10238): 1705-1714

32. Mancia G, Rea F, Ludergnani M, Apolone G, Corrao G. Reninangiotensin-aldosterone system blockers and the risk of COVID-19. $\mathrm{N}$ Engl J Med 2020; [Epub ahead of print] doi: 10.1056/ NEJMoa2006923

33. Singer M, Deutschman CS, Seymour CW, Shankar-Hari M, Annane D, Bauer M, Bellomo R, Bernard GR, Chiche JD, Coopersmith CM, Hotchkiss RS, Levy MM, Marshall JC, Martin GS, Opal SM, Rubenfeld GD, van der Poll T, Vincent JL, Angus DC. The third international consensus definitions for sepsis and septic shock (sepsis-3). JAMA 2016; 315(8): 801-810

34. Force ADT, Ranieri VM, Rubenfeld GD, Thompson BT, Ferguson ND, Caldwell E, Fan E, Camporota L, Slutsky AS. Acute respiratory distress syndrome: the Berlin Definition. JAMA 2012; 307(23): 2526-2533

35. KDIGO. Clinical Practice Guideline for Acute Kidney Injury. 2012. https://kdigo.org/wp-content/uploads/2016/10/KDIGO-2012-AKIGuideline-English.pdf (accessed May 24, 2020)

36. Huang C, Wang Y, Li X, Ren L, Zhao J, Hu Y, Zhang L, Fan G, Xu J, Gu X, Cheng Z, Yu T, Xia J, Wei Y, Wu W, Xie X, Yin W, Li H, Liu M, Xiao Y, Gao H, Guo L, Xie J, Wang G, Jiang R, Gao Z, Jin Q, Wang J, Cao B. Clinical features of patients infected with 2019 novel coronavirus in Wuhan, China. Lancet 2020; 395(10223): 497506

37. Abe M, Oikawa O, Okada K, Soma M. Urinary angiotensinconverting enzyme 2 increases in diabetic nephropathy by angiotensin II type 1 receptor blocker olmesartan. J Renin Angiotensin Aldosterone Syst 2015; 16(1): 159-164

38. Furuhashi M, Moniwa N, Mita T, Fuseya T, Ishimura S, Ohno K, Shibata S, Tanaka M, Watanabe Y, Akasaka H, Ohnishi H, Yoshida H, Takizawa H, Saitoh S, Ura N, Shimamoto K, Miura T. Urinary angiotensin-converting enzyme 2 in hypertensive patients may be increased by olmesartan, an angiotensin II receptor blocker. Am J Hypertens 2015; 28(1): 15-21

39. Soro-Paavonen A, Gordin D, Forsblom C, Rosengard-Barlund M, Waden J, Thorn L, Sandholm N, Thomas MC, Groop PH; FinnDiane Study Group. Circulating ACE2 activity is increased in patients with type 1 diabetes and vascular complications. J Hypertens 2012; 30(2): 375-383

40. Liu J, Ji H, Zheng W, Wu X, Zhu JJ, Arnold AP, Sandberg K. Sex differences in renal angiotensin converting enzyme 2 (ACE2) activity are $17 \beta$-oestradiol-dependent and sex chromosome-independent. Biol Sex Differ 2010; 1(1): 6

41. Ferrario CM, Jessup J, Gallagher PE, Averill DB, Brosnihan KB, Ann Tallant E, Smith RD, Chappell MC. Effects of reninangiotensin system blockade on renal angiotensin-(1-7) forming enzymes and receptors. Kidney Int 2005; 68(5): 2189-2196

42. Bunyavanich S, Do A, Vicencio A. Nasal gene expression of angiotensin-converting enzyme 2 in children and adults. JAMA 2020; [Epub ahead of print] doi: 10.1001/jama.2020.8707

43. Schouten LR, van Kaam AH, Kohse F, Veltkamp F, Bos LD, de Beer FM, van Hooijdonk RT, Horn J, Straat M, Witteveen E, Glas GJ, Wieske L, van Vught LA, Wiewel MA, Ingelse SA, Cortjens B, van Woensel JB, Bos AP, Walther T, Schultz MJ, Wosten-van Asperen RM; MARS consortium. Age-dependent differences in pulmonary host responses in ARDS: a prospective observational cohort study. Ann Intensive Care 2019; 9(1): 55

44. Imai Y, Kuba K, Rao S, Huan Y, Guo F, Guan B, Yang P, Sarao R, Wada T, Leong-Poi H, Crackower MA, Fukamizu A, Hui CC, Hein L, Uhlig S, Slutsky AS, Jiang C, Penninger JM. Angiotensinconverting enzyme 2 protects from severe acute lung failure. Nature 2005; 436(7047): 112-116

45. Gheblawi M, Wang K, Viveiros A, Nguyen Q, Zhong JC, Turner AJ, Raizada MK, Grant MB, Oudit GY. Angiotensin-converting enzyme 2: SARS-CoV-2 receptor and regulator of the reninangiotensin system: celebrating the 20th anniversary of the discovery of ACE2. Circ Res 2020; 126(10): 1456-1474 
46. Gu A, Farzadeh SN, Chang YJ, Kwong A, Lam S. Patterns of antihypertensive drug utilization among US Adults with diabetes and comorbid hypertension: the national health and nutrition examination survey 1999-2014. Clin Med Insights Cardiol 2019; 13: 1179546819839418

47. Joint Committee for Guideline Revision. 2018 Chinese Guidelines for Prevention and Treatment of Hypertension-A report of the Revision Committee of Chinese Guidelines for Prevention and Treatment of Hypertension. J Geriatr Cardiol 2019; 16(3): 182-241

48. Meng J, Xiao G, Zhang J, He X, Ou M, Bi J, Yang R, Di W, Wang Z, Li Z, Gao H, Liu L, Zhang G. Renin-angiotensin system inhibitors improve the clinical outcomes of COVID-19 patients with hypertension. Emerg Microbes Infect 2020; 9(1): 757-760

49. Peng YD, Meng K, Guan HQ, Leng L, Zhu RR, Wang BY, He MA, Cheng LX, Huang K, Zeng QT. Clinical characteristics and outcomes of 112 cardiovascular disease patients infected by 2019nCoV. Chin J Cardiol (Zhonghua Xin Xue Guan Bing Za Zhi) 2020; 48(0): E004

50. Bavishi C, Maddox TM, Messerli FH. Coronavirus disease 2019 (COVID-19) infection and renin angiotensin system blockers. JAMA Cardiol 2020; [Epub ahead of print] doi: 10.1001/ jamacardio.2020.1282 